\section{Amy R. Guest \\ Peter J. Strouse \\ Chee Chung Hiew \\ Marjorie Arca}

\title{
Progressive esophageal leiomyomatosis with respiratory compromise
}

Received: 16 August 1999

Accepted: 25 October 1999

A. R. Guest · P. J. Strouse (

Section of Pediatric Radiology - F3503,

C.S. Mott Children's Hospital,

Department of Radiology,

University of Michigan Health System,

Ann Arbor, MI 48109-0252, USA

\section{C.C.Hiew}

Department of Pediatric Imaging,

Detroit Medical Center,

Wayne State University,

Children's Hospital of Michigan,

Detroit, Michigan, USA

M. Arca

Section of Pediatric Surgery,

C.S. Mott Children's Hospital,

Department of Surgery,

University of Michigan Health System,

Ann Arbor, Michigan, USA

\author{
Abstract Leiomyomatosis is a rare \\ neoplastic condition of the pediatric \\ esophagus. Presenting symptoms \\ usually overlap with more common \\ esophageal disorders, namely, gas- \\ troesophageal reflux. A patient is \\ presented in whom leiomyomatosis \\ progressed to the point of causing \\ cachexia and respiratory compro- \\ mise.
}

\section{Introduction}

Esophageal leiomyomatosis is a rare condition causing leiomyomatous thickening of all or part of the esophagus. It has been diagnosed in patients as young as 6 months of age and as old as 75 years, but is predominantly a disease of younger patients [1-4]. Esophageal leiomyomatosis is associated with Alport syndrome and multiorgan leiomyomatosis syndromes [1, 5-7]. Patients with esophageal leiomyomatosis usually present with progressive dysphagia and other associated nonspecific gastrointestinal complaints, including weight loss, vomiting, and epigastric pain $[1,4,8]$. Treatment includes esophagectomy. We present an unusual case of esophageal leiomyomatosis, with progression of disease to the point of cachexia and respiratory compromise.

\section{Case report}

A 13-year-old girl presented to our institution with a fever of $105^{\circ} \mathrm{F}$, mild respiratory distress, and epigastric pain. A history of long-standing gastroesophageal reflux, esophageal dysmotility, and multiple hospitalizations for asthma and pneumonia was initially provided. The patient had no history or clincial evidence of renal, eye, or ear disease. No gynecologic or anorectal abnormality was known.

Physical examination at the time of admission revealed a cachectic child who was small for her age. The patient was wheezing and had sternal retractions. A dilated esophagus was suspected based on the admission chest radiograph (Fig.1). The lungs were hyperinflated. An upper gastrointestinal examination was attempted; however, the child could not lie supine and drink barium due to respiratory symptoms and the examination was aborted. Chest computed tomography (CT) was requested to characterize the suspected lung disease better. The CT confirmed lung hyperexpansion 

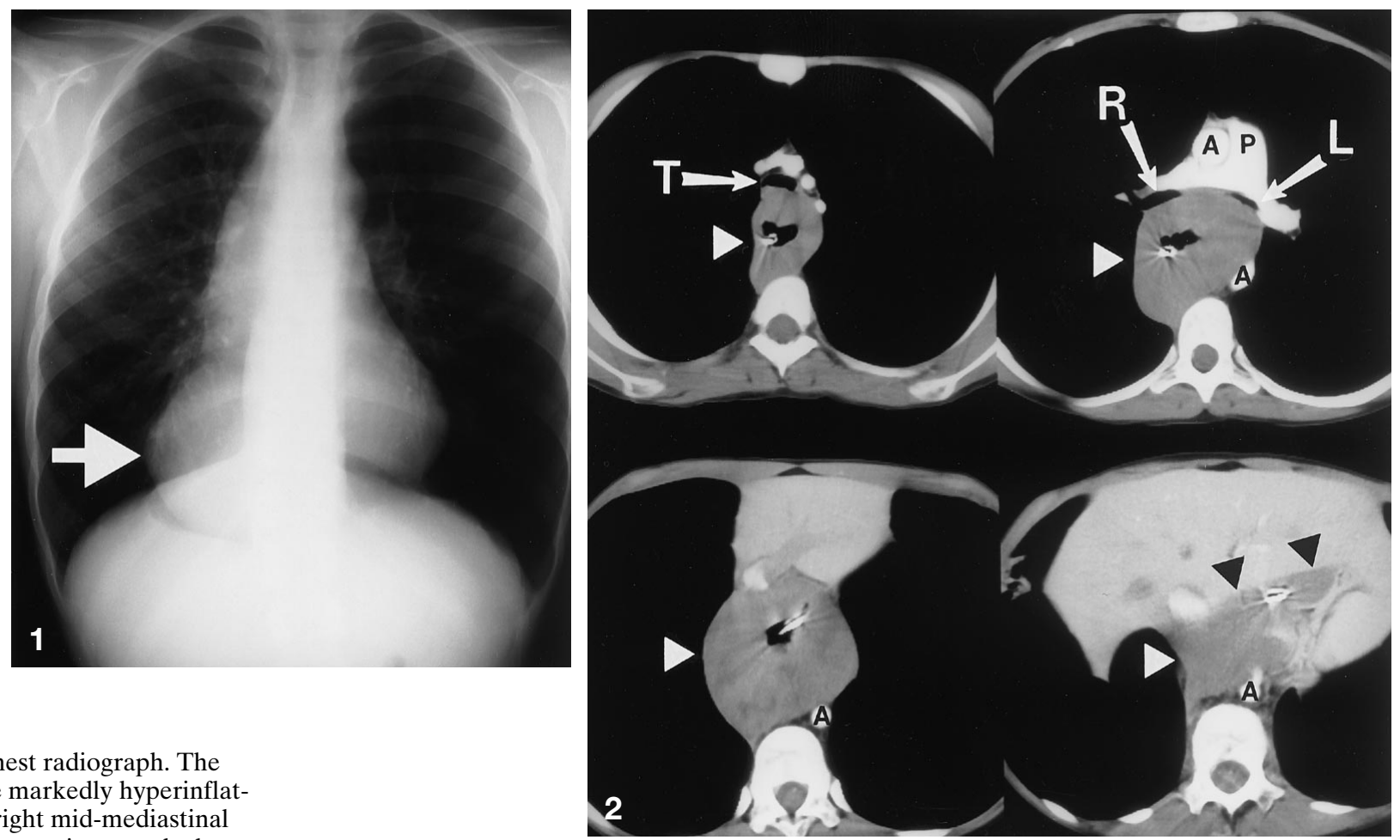

Fig. 1 Chest radiograph. The lungs are markedly hyperinflated. The right mid-mediastinal border is prominent and a lower mediastinal mass is noted (arrow)

Fig. 2 Computed tomography (1998). The esophageal wall is markedly thickened (white arrowheads), particularly in the distal esophagus. A nasogastric tube is present in the esophageal lumen. The distal trachea (T) and proximal mainstem bronchi ( $R$ right, $L$ left) are narrowed. The abnormality extends into proximal stomach (black arrowheads). ( $A$ aorta, $P$ main pulmonary artery)

Fig.3 Computed tomography (1988). Although the esophageal wall is diffusely thickened (arrowheads), the degree of abnormality is substantially less than in 1998 (Fig. 2), indicating interval progression. ( $A$ aorta, $T$ trachea, $R$ right, $L$ left mainstem bronchi)

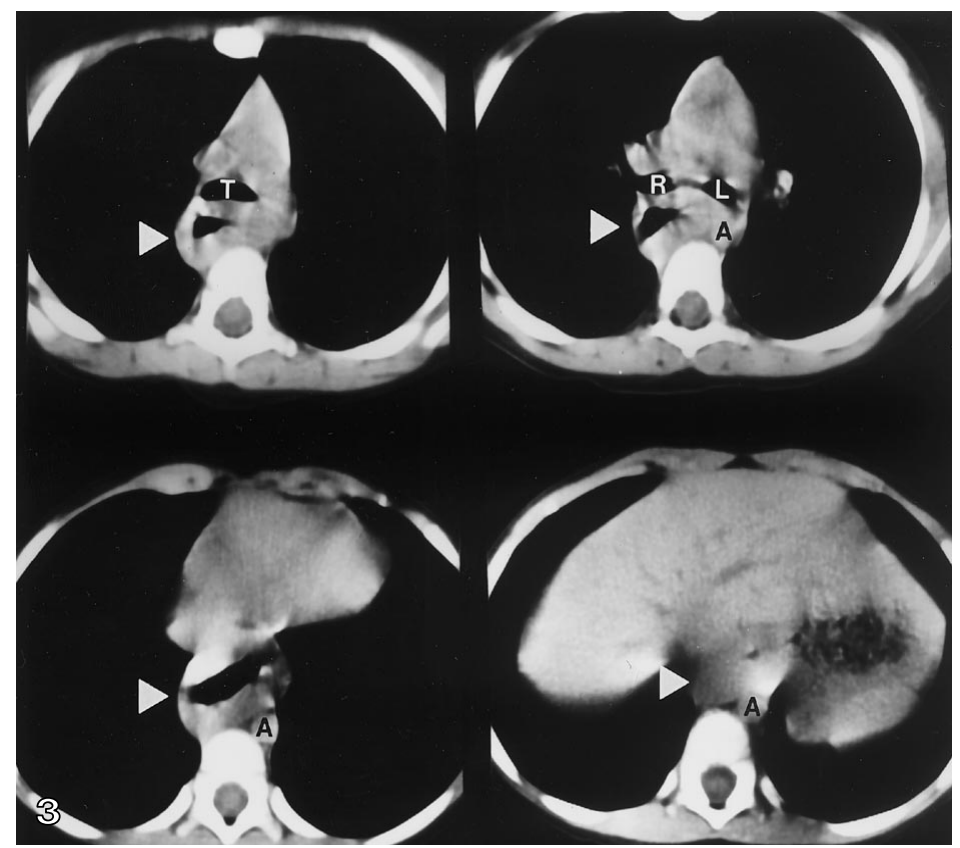

and showed non-specific patchy air space opacities in the lung bases. Diffuse esophageal wall thickening was seen extending from the thoracic inlet to the gastroesophageal junction and gastric cardia (Fig. 2). Maximal esophageal wall thickness was $5 \mathrm{~cm}$. The esophageal mass caused extrinsic narrowing and displacement of the distal trachea and proximal bronchi.

After the esophageal abnormality was delineated, further history was obtained. It was determined that the child had been treat- ed at a neighboring institution for approximately 10 years. The esophageal abnormality had been well documented previously by upper gastrointestinal examination, CT (Fig. 3), and magnetic resonance imaging. The mass had progressed considerably in size over the 10-year period since the patient's initial CT. Although the diagnosis of leiomyomatosis had been suggested on the basis of these studies, and surgery recommended, the patient's family had refused surgical therapy. 


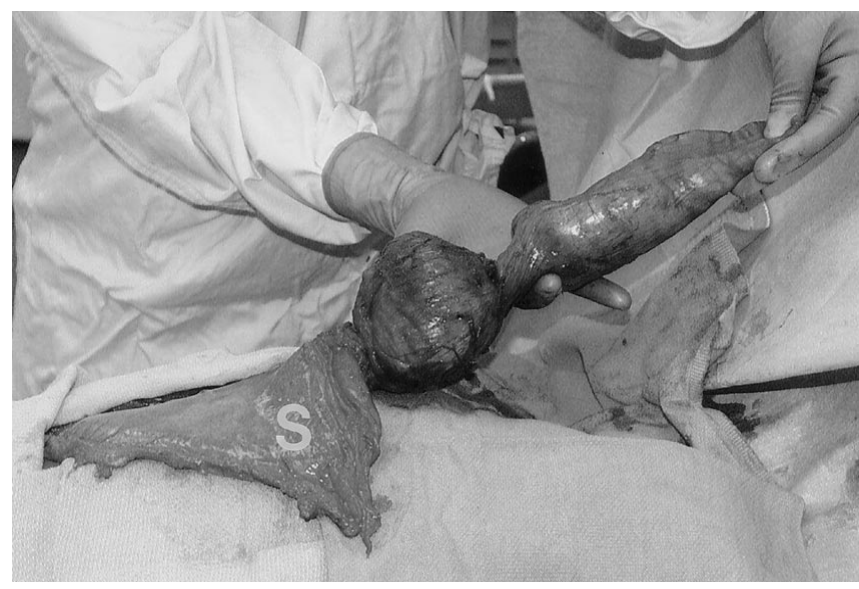

Fig. 4 Intraoperative photograph of partially resected esophagus. ( $S$ stomach)

Esophagoscopy and bronchoscopy were performed. Examination of the esophagus showed predominantly normal mucosa with minimal inflammatory change. The esophageal mass was inapparent. When bronchoscopy was attempted, the patient's left lung completely collapsed. The patient's respiratory status temporarily decompensated, nearly requiring cardiopulmonary resuscitation. The procedure was aborted. After 2 weeks of total parenteral nutrition and antibiotic therapy for presumed pneumonia, the patient underwent esophagectomy and gastric pull-through. The resected esophageal specimen was noted to contain multiple lobules with gross overall thickening of the wall (Fig. 4). Gross pathologic evaluation revealed nearly circumferential hypertrophy of the muscularis propria of varying thickness indicative of esophageal leiomyomatosis. Following surgery, the patient's respiratory symptoms improved considerably, and to date she has had no further admissions for asthma or pneumonia.

\section{Discussion}

Although quite rare, leiomyoma is the most common benign tumor of the esophagus. Children comprise only $2.6 \%$ of patients with documented leiomyomas [7]. In children, lesions are more likely to be multiple or diffuse than in adults. Leiomyomas in children are also more likely to be associated with Alport syndrome or multiple organ leiomyomatosis syndromes [7]. In esophageal leiomyomatosis, the esophagus is studded with multiple leiomyomas. The involved esophagus demonstrates marked circumferential wall thickening. The abnormality is most commonly found in the distal third of the esophagus, but can affect the entire length of the esophagus and also extend into the proximal stomach. Both the circular and the longitudinal muscle layers may be involved. Microscopically, the lesions appear as typical leiomyomas, consisting of interleaving spindle cell fascicles, without cellular atypia or mitoses. Fibrous tissue is sometimes present, often in large amounts, and myomatous transformation of blood ves- sels and hypertrophy of involved nerves has been reported $[1,2,8]$.

The average age at presentation and gender distribution of esophageal leiomyomatosis is unclear. While studies typically identify esophageal leiomyomatosis as a disease of younger people, earlier literature reported patient presenting age as ranging from 17 to 75 years with a 3:1 male predominance $[2,8]$. More recent series identify esophageal leiomyomatosis as presenting in a much younger population (average age of 11 years), with a slight female predominance $[1,6]$. The recent series, however, include predominantly patients with Alport syndrome or other familial esophageal disease, which may contribute to the earlier development of lesions and earlier presentation.

Patients with esophageal leiomyomatosis most often present with slow, progressive dysphagia, which can mimic gastroesophageal reflux or an esophageal dysmotility disorder. Chest pain, vomiting, regurgitation, epigastric pain, and weight loss are common presenting complaints $[1,4,8]$. On plain radiographs, rightward deviation of the azygoesophageal stripe and retrotracheal soft tissue has been described [1]. Barium studies frequently demonstrate smooth, tapered narrowing of the distal esophagus with diminished peristalsis. Esophageal wall thickening may be apparent. Filling defects in the gastric fundus caused by the esophageal mass or contiguous gastric involvement have also been reported $[1,8,9]$. Superficial biopsy on endoscopic examination is unrewarding, usually yielding a diagnosis of esophagitis [7].

The differential diagnosis of a diffusely thickened esophageal wall in a child is limited. Leiomyosarcoma, lymphoma, and esophageal carcinoma of the pediatric esophagus are all very rare. While a benign inflammatory process such as esophagitis and chronic granulomatous disease may cause mild esophageal wall thickening, the degree of wall thickening is much less than that seen in our patient.

Achalasia causes esophageal dilatation and might produce a chest radiographic appearance of esophageal mass effect similar to that seen in our patient; however, the esophageal wall is not substantially thickened. Primary achalasia and esophageal leiomyomatosis do have a similar clinical presentation. Many patients with leiomyomatosis carry a diagnosis of achalasia incorrectly until their leiomyomatosis is diagnosed. The two entities may have a similar appearance on barium studies. Primary achalasia usually affects a shorter segment of the esophagus than esophageal leiomyomatosis and does not affect the gastric cardia [1]. CT is helpful in differentiating the two disorders by assessing the degree of esophageal wall thickening present.

Idiopathic muscular hypertrophy can appear similar to mild esophageal leiomyomatosis on radiographic studies. Patients with idiopathic muscular hypertrophy 
tend to be older than those with esophageal leiomyomatosis, with the average age at presentation reported in some studies to be as late as the 6th decade of life [10]. A maximum wall thickness of $15 \mathrm{~mm}$ has been reported and the cardia is rarely involved, further differentiating idiopathic muscular hypertrophy from esophageal leiomyomatosis $[1,11]$.

Although leiomyomatosis is not known to undergo malignant degeneration, treatment generally consists of esophagectomy and gastric pull-through for functional reasons $[1,2]$. Myotomy or enucleation of lyomyomas is not successful in these patients due to the diffuse nature of disease $[3,4,8]$.

Our patient is unusual in that her esophageal leiomyomatosis progressed to the extent that it caused respira- tory symptoms. The development of these symptoms was secondary to gradual progression of esophageal disease and the location of leiomyomas, adjacent to the airway, causing compression the carina. Prior reports of this degree of respiratory compromise due to leiomyomatosis are rare [6]. Even in advanced cases, the overwhelming majority of reported deaths from leiomyomatosis have been secondary to advanced malnutrition, not respiratory distress $[2,3]$.

Esophageal leiomyomatosis is a rare cause of marked esophageal wall thickening. It should be considered in a child with prolonged dysphagia and esophageal mass. Although respiratory symptoms are not commonly encountered, they can develop with advanced disease due to airway compression.

\section{References}

1. Levine MS, Buck JL, PantongragBrown L, et al (1996) Esophageal leiomyomatosis. Radiology 199: 533-536

2. Heald J, Moussalli H, Haselton PS (1986) Diffuse leiomyomatosis of the oesophagus. Histopathology 10: 755-759

3. Muthuphei NM (1998) Diffuse leiomyomatosis of the oesophagus - a case report. S African J Surg 36: 30-31

4. Lonsdale RN, Roberts PF, Vaughan R, et al (1992) Familial oesophageal leiomyomatosis and nephropathy. Histopathology 20: 127-133
5. Lerone M, Dodero P, Romeo G, et al (1991) Leiomyomatosis of oesophagus, congenital cataracts and hematuria. Pediatr Radiol 21: 578-579

6. Cochat P, Guibaud P, Torres R, et al (1998) Diffuse leiomyomatosis in Alport syndrome. J Pediatr 113: 339-343

7. Rabushka LS, Fishman EK, Kuhlman JE, et al (1991) Diffuse esophageal leiomyomatosis in a patient with Alport syndrome: CT demonstration. Radiology 179: 176-178

8. Fernandes JP, Mascarenhas MJ, da Costa JC, et al (1975) Diffuse leiomyomatosis of the esophagus. Digestive Diseases 20: 684-690
9. Schapiro RL, Sandrock AR (1973) Esophagogastric and vulvar leiomyomatosis: a new radiologic syndrome. J Can Assoc Radiol 24: 184-187

10. Agostini S, Grimaud JC, Salducci J, et al (1988) Idiopathic muscular hypertrophy of the esophagus: CT features. JCAT 12: 1041-1043

11. Kreczy A, Gassner J, Mikuz G (1990) Idiopathic hypertrophy of the oesophagus in children. Virchows Arch 417: 81-84 